\title{
Synthesizing a human-like voice is the easy way
}

\author{
Sébastien Le Maguer \\ lemagues@tcd.ie \\ ADAPT Centre, Trinity College Dublin \\ Dublin, Ireland
}

\begin{abstract}
Deep-learning based technologies produce speech that is almost indistinguishable from humans. However, focusing on producing human-like voices poses ethical, security and societal issues. Considering the flexibility and the regression power of new technologies based on deep-learning, it is now time to consider a new type of synthesis: natural non-human-like speech synthesis. This paper aims to convince you that such research opens new research directions, that it brings another perspective to address human-like speech challenges, and that enough material is available to start to investigate non-human-like speech.
\end{abstract}

\section{CCS CONCEPTS}

•Human-centered computing $\rightarrow$ Natural language interfaces.

\section{KEYWORDS}

Speech synthesis, human-like/non-human-like sounding voice

\section{ACM Reference Format:}

Sébastien Le Maguer and Benjamin R. Cowan. 2021. Synthesizing a humanlike voice is the easy way. In $3 r d$ Conference on Conversational User Interfaces (CUI '21), July 27-29, 2021, Bilbao (online), Spain. ACM, New York, NY, USA, 3 pages. https://doi.org/10.1145/3469595.3469614

\section{INTRODUCTION}

Thanks to the human-like quality of modern Text-To-Speech (TTS) technologies, Voice User Interface (VUI) has now become commonly used in everyday life application. The release of WaveNet[22] and the subsequent systems, such as Tacotron[18], kicked off this paradigm shift and led the big tech companies to invest in voice technologies massively. Consequently, the speech synthesis community currently focuses on two complementary axes to enrich voices used in VUI: providing a better control of the TTS systems; improving the expressivity of the produced synthetic speech.

The primary assumption of numerous recently published research studies in speech synthesis is that natural speech is synonymous with human-like speech. While producing human-sounding speech is one important direction to investigate, we argue that focusing the research only to reach this holy grail is counter-productive. In order to prove it, we present three key arguments:

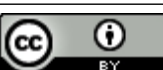

This work is licensed under a Creative Commons Attribution International 4.0 License.

CUI '21, fuly 27-29, 2021, Bilbao (online), Spain

(c) 2021 Copyright held by the owner/author(s).

ACM ISBN 978-1-4503-8998-3/21/07.

https://doi.org/10.1145/3469595.3469614

\author{
Benjamin R. Cowan \\ benjamin.cowan@ucd.ie \\ ADAPT Centre, University College Dublin \\ Dublin, Ireland
}

(1) by focusing exclusively on human-like speech, research amplifies ethical issues and ignore some important opportunities for VUI and beyond;

(2) producing non-human-like speech addresses challenges which are still under-investigated in speech science and speech technology;

(3) some directions already exist to produce non-human-like speech.

\section{ARGUMENT 1: ETHICAL CONCERNS AND MISSED OPPORTUNITIES}

The primary ethical concerns about the rendering of human-like speech is "voice spoofing". It consists of an entity (human or computer) usurping the identity of an other human. The most wellknown spoofing system is DeepFake[14] and, while it is focusing mainly on video, the results of these systems give an idea of the capabilities of such technologies. As pointed by the spoofing challenge organizers, TTS poses a great spoofing threat and it can have real life consequences ${ }^{1}$. While spoofing detection is highly active research field [20], speech synthesis researchers and technologists have to be aware about such impact on make synthetic speech more and more human-sounding.

Focusing on VUI, a UNESCO report [24] pointed out that the use of female human-sounding voice let users to "bully" the device. This show that using human-like voice can also have a societal impact aside securities. While this report focuses on gender equality, it is important to also take into account that speech carries more diverse information about the person. Accents and linguistic codes, among others, gives information about the ethnicity and the social class of the speaker. Therefore, using a human-like voice in a device is likely to reinforce some stereotypes.

All these issues are known and considered important topics in Conversational User Interface (CUI) [5]. However, aside these serious concerns, focusing on human-sounding speech synthesis also considerably limits the potential of such technology. If we consider the entertainment industry or robots, generating non-human speech can be a game changer. It could bring a new dimension to non-human characters which are presents in movie such as fantasy or science-fiction movies [2]. It could also provide robots a proper identity as it has been discussed in [13].

In addition, producing human-like voice could be problematic for the intelligibility of the message conveyed. For example, if we assume a noisy environment, producing a speech which ignores human physiology constraints could lead to a better intelligibility by exaggerating the prominence of some frequencies. For now, speech enhancement mainly consists of post-processing the speech

${ }^{1}$ see https://www.wsj.com/articles/fraudsters-use-ai-to-mimic-ceos-voice-in-unusualcybercrime-case- 11567157402 
signal to remove the noise [17] or by training voices using different speaking styles [9].

Finally, it is important to remember that synthetic speech is a crucial empowerment technology for visually impaired people. However, their use of synthetic speech differs drastically from the default use case defined for VUI. Ultra-fast speech[15] is a way for blind people to "browse" the information. While it is not intelligible for the targeted population of standard smart speakers, investigating the production of such speech while still maintaining the key components of the message will be key for visually impaired people to make the most of VUI systems.

To conclude this part, we see that producing non-human-like speech provides numerous opportunities which can't be ignored. While this is a complicated matter which forces the community to face complicated challenges, the next section will prove that these challenges will have to be addressed even for human-like synthetic speech.

\section{ARGUMENT 2: NON-HUMAN-LIKE SPEECH SYNTHESIS CHALLENGES ARE SPEECH SYNTHESIS CHALLENGES}

Historically, the focus of speech synthesis research was on the production of a highly intelligible speech signal. Lately, this focus moved from intelligibility to naturalness as the quality of synthetic speech drastically improved due to the development of new technologies. However, natural speech is still considered synonymous with human-like speech. This reduction can be partially blamed on the lack of proper speech synthesis analysis methodologies.

The currently used standard subjective evaluation methodologies rely on considering the speech signal out of context. Participants are required to listen to utterances and either score $[10,11]$ them either choose their preferred signal [6] among a randomized combination of synthetic signals. More recent protocols evaluate the cognitive load entailed by a synthesis methodology using pupillometry [7] or EEG [16].

These protocols showed that using parametrical speech synthesis led to a higher cognitive load which can be explained by the lack of variability and the signal's buzziness [8]. However, because of the limitation of the investigated technologies, it is difficult to pinpoint the source of this phenomenon. To understand more deeply how speech is perceived, researchers will have to be able to decompose with a higher precision the speech signal. Investigating non-humanlike speech can lead to finding ways to achieve such decomposition as it implies to study the potential disentanglement of the voice characteristics from the speech patterns.

An even more crucial problem to develop non-human-like speech synthesis is how to evaluate this kind of signals. As we have seen, the use of the current protocols will provide limited information about the nature of non-human-like speech. Indeed such a speech signal is likely to be disconcerting at first glance. This implies that evaluation methodologies will have to consider how it is easy for listeners to get accustomed to the produced speech.

While it seems an additional issue, several studies have already shown that context is critical for fully appreciating the quality of a synthetic speech type. [21] showed that there should be a congruence between the physical aspect of the robot and its voice quality;
[4] showed that the context when the evaluation is taking place also has a significant impact on the results. In [23], future directions and scenarios to evaluate human-like synthetic speech have been discussed. These scenarios are also an appropriate starting point to evaluate non-human-like speech signals. However, an initial learning phase will be necessary as non-human-like speech is not yet part of our everyday life.

To conclude this section, we saw that non-human-like speech synthesis will face two critical challenges: how to disentangle the voice component from the speech component and how to evaluate such signal. We also saw that human-like speech synthesis also have to face these challenges. From these, we can deduce that any progress achieved in one of the challenges is likely to provide insights to face the other one. However, in comparison to its counterpart, non-human-like speech synthesis is a new direction, and, therefore, we need starting material.

\section{ARGUMENT 3: WE KNOW WHERE TO START THIS JOURNEY}

Historical technologies, such as formant synthesis or even HMM synthesis, are able to synthesize speech identified as "machine-like speech". From this, we can deduce that we, humans, are open to the idea of non-human-like speech. However, as we have already seen, the quality of parametrical synthetic speech is not satisfying as listening to it requires a high cognitive load. As we have also seen, we are not yet able to understand what causes this load. While more perception studies are needed to explore the reasons behind this high cognitive load, other research areas are already investigating ways to manipulate an audio signal to reach uncharted territories. Indeed, contemporary music and research in psycho-acoustic are at the forefront of timbre manipulation and analysis.

In pop culture, auto-tune [1] is commonly used to modify voice timbre. Its robotic aspect is already accepted as an artistic component ${ }^{2}$. Music technology is also fastly evolving, and new instruments provide new ways of filtering the voice at the recording stage. The Pipe from SOMA [19], and especially the contact microphone developed for this instrument, is a typical example of such technologies. Finally, even if they are not focusing on speech directly, contemporary music performers and composers are already exploring new territories about what is sound and what can the human apparatus can produce[3]. We should consider these experimentations as an opportunity to reflect on how to think about the speech signal and pushing the boundaries of what is possible now that we can disregard the physiological constraints of speech production.

Another angle of approach is to consider how Machine Learning (ML) is used to move forward the creation of new sound. As a representative example, we can cite the project Magenta from Google, and more specifically NSynth Super ${ }^{3}$. NSynth Super relies on a deep learning approach to morph the timbre of multiple instruments to create a type of instruments. This project is a key example of the potential symbiosis between ML and timbre modifications. Aside from the auto-tune example, all of these works do not rely

\footnotetext{
${ }^{2}$ The informal term "Cher Effect", associated with the specific use of the auto-tune technology with extreme parameters in the song Believe of the singer Cher, is representative of the acceptation of such aspects

${ }^{3}$ https://nsynthsuper.withgoogle.com/
} 
on speech. Yet, they show us different directions in order to design a non-human-like speech.

Transposing the idea of hybridizing instrument timbre to create a new sound, we could rely on chimeric speech to provide an equivalent for speech. While not applied to produce synthetic speech, this technic is already used in speech perception and speech intelligibility. Consequently, combined with neural technologies, investigating chimeric speech could provide a starting point to generate non-human-like speech which is still intelligible and does not cause fatigue.

Finally, as we have mentioned in the previous section, evaluation remains a critical issue. Based on the previous paragraph, we also saw that timbre modification would be essential to develop nonhuman-like speech. Therefore, we also need to think about ways of analyzing precisely the timbre characteristics of the speech.

Studies in psych-acoustic already provide tools to analyze timbre. The thesis of Megan L. Lavengood[12] proposes a methodology rooted in two fundamental components of the perception of the timbre in music: the description of the signal via spectrograms; analyzing how the cultural context influences the perception of the listeners. While this methodology focuses on 80 s popular music, it shows a different way to categorize sounds and conduct analysis of such categorization.

\section{FINAL STATEMENT}

In this paper, we did not talk about the advantages of producing human-like speech. They are numerous, and they should not be neglected. However, we showed that, while investigating the production of non-human-like speech is a challenging direction, it opens a new world of opportunities. Firstly, It will force researchers to define precisely essential concepts such as naturalness. It can also open a new way to deal more responsibly with voices and address some challenges such as voice privacy. Finally, it may provide new ways to build VUIs which are more adapted to their environment.

Finally, we have also seen that we have the tools to start conducting such research. Therefore, to conclude this paper, the only remaining comment about non-human-like speech synthesis we can do is that we have to start doing it.

\section{ACKNOWLEDGMENTS}

The authors would like to thank Dr Ann Cleare for eyes opening discussions about contemporary music and modern music devices. This research was conducted with the financial support of Irish Research Council (IRC) under Grant Agreement No. 208222/15425 at the ADAPT SFI Research Centre at Trinity College Dublin. ADAPT, the SFI Research Centre for AI-Driven Digital Content Technology, is funded by Science Foundation Ireland through the SFI Research Centres Programme under Grant No. 13/RC/2106_P2.

\section{REFERENCES}

[1] Antares Audio. 2021. AUTO-TUNE PRO - The Professional Standard for Pitch Correction. https://www.antarestech.com/product/auto-tune-pro [Online; accessed 12. Apr. 2021].

[2] Matthew P. Aylett, Selina Jeanne Sutton, and Yolanda Vazquez-Alvarez. 2019 The Right Kind of Unnatural: Designing a Robot Voice. In Proceedings of the 1st International Conference on Conversational User Interfaces (Dublin, Ireland) (CUI '19). Association for Computing Machinery, New York, NY, USA, Article 25, 2 pages. https://doi.org/10.1145/3342775.3342806
[3] Gelsey Bell. 2019. Gestural Song Form in Experimental Vocal Music. Performance Research 24, 1 (Jan. 2019), 45-52. https://doi.org/10.1080/13528165.2019.1593735 [4] Simon Betz, Birte Carlmeyer, Petra Wagner, and Britta Wrede. 2018. Interactive Hesitation Synthesis: Modelling and Evaluation. Multimodal Technologies and Interaction 2, 1 (2018). https://doi.org/10.3390/mti2010009

[5] Heloisa Candello, Cosmin Munteanu, Leigh Clark, Jaisie Sin, María Inés Torres, Martin Porcheron, Chelsea M. Myers, Benjamin Cowan, Joel Fischer, Stephan Schlögl, Christine Murad, and Stuart Reeves. 2020. CUI@CHI: Mapping Grand Challenges for the Conversational User Interface Community. In Extended $A b$ stracts of the 2020 CHI Conference on Human Factors in Computing Systems (Honolulu, HI, USA) (CHI EA '20). Association for Computing Machinery, New York, NY, USA, 1-8. https://doi.org/10.1145/3334480.3375152

[6] David Clark. 1982. High-resolution subjective testing using a double-blind comparator. Fournal of the Audio Engineering Society 30, 5 (1982), 330-338.

[7] Avashna Govender and Simon King. 2018. Measuring the Cognitive Load of Synthetic Speech Using a Dual Task Paradigm.. In INTERSPEECH. 2843-2847.

[8] Avashna Govender, Cassia Valentini-Botinhao, and Simon King. 2019. Measuring the contribution to cognitive load of each predicted vocoder speech parameter in dnn-based speech synthesis. In Submitted to Speech Synthesis Workshop (SSW), Vol. 2019.

[9] Q. Hu, T. Bleisch, P. Petkov, T. Raitio, E. Marchi, and V. Lakshminarasimhan. 2021. Whispered and Lombard Neural Speech Synthesis. In IEEE Spoken Language Technology Workshop (SLT). 454-461. https://doi.org/10.1109/SLT48900.2021. 9383454

[10] ITU. 1996. Methods for subjective determination of transmission quality. ITU-T Recommendation P.800. International Telecommunication Union (ITU-P), Geneva.

[11] ITU. 2001. Method for the Subjective Assessment of Intermediate Sound Quality (MUSHRA). Technical Report P.1534-1. International Telecommunication Union (ITU-R).

[12] Megan Lavengood. 2017. A New Approach to the Analysis of Timbre. Ph.D. Dissertation. http://dx.doi.org/10.17613/M6FG5W.

[13] Sébastien Le Maguer. 2021. Will synthetic speech provide a suitable voice for robots?. In Robo-Identity: Artificial identity and multi-embodiment.

[14] Yaniv Leviathan and Yossi Matias. 2018. Google Duplex: An AI System for Accomplishing Real-World Tasks Over the Phone. https://ai.googleblog.com/ 2018/05/duplex-ai-system-for-natural-conversation.html

[15] Anja Moos and Jürgen Trouvain. 2007. Comprehension of Ultra-Fast SpeechBlind vs.'Normally Hearing'Persons. In Proceedings of the 16th International Congress of Phonetic Sciences, Vol. 1. Saarland University Saarbrücken, Germany, 677-680.

[16] Ivan Halim Parmonangan, Hiroki Tanaka, Sakriani Sakti, Shinnosuke Takamichi, and Satoshi Nakamura. 2019. Speech Quality Evaluation of Synthesized Japanese Speech Using EEG. In Proc. Interspeech 2019. 1228-1232. https://doi.org/10.21437/ Interspeech.2019-2059

[17] Jan Rennies, Henning Schepker, Cassia Valentini-Botinhao, and Martin Cooke. 2020. Intelligibility-Enhancing Speech Modifications - The Hurricane Challenge 2.0. In Proc. Interspeech 2020. 1341-1345. https://doi.org/10.21437/Interspeech. 2020-1641

[18] Jonathan Shen, Ruoming Pang, Ron J. Weiss, Mike Schuster, Navdeep Jaitly, Zongheng Yang, Zhifeng Chen, Yu Zhang, Yuxuan Wang, Rj Skerrv-Ryan, Rif A. Saurous, Yannis Agiomvrgiannakis, and Yonghui Wu. 2018. Natural TTS Synthesis by Conditioning Wavenet on MEL Spectrogram Predictions. 2018 IEEE International Conference on Acoustics, Speech and Signal Processing (ICASSP) (2018), 15-20. https://doi.org/10.1109/ICASSP.2018.8461368

[19] SOMA LABORATORY. 2021. The Pipe. https://somasynths.com/pipe [Online; accessed 15. Apr. 2021].

[20] Massimiliano Todisco, Xin Wang, Ville Vestman, Md. Sahidullah, Héctor Delgado, Andreas Nautsch, Junichi Yamagishi, Nicholas Evans, Tomi H. Kinnunen, and Kong Aik Lee. 2019. ASVspoof 2019: Future Horizons in Spoofed and Fake Audio Detection. In Proc. Interspeech 2019. 1008-1012. https://doi.org/10.21437/ Interspeech.2019-2249

[21] Ilaria Torre, Jeremy Goslin, Laurence White, and Debora Zanatto. 2018. Trust in Artificial Voices: A "Congruency Effect" of First Impressions and Behavioural Experience. Association for Computing Machinery, New York, NY, USA. https: //doi.org/10.1145/3183654.3183691

[22] Aäron Van den Oord, Sander Dieleman, Heiga Zen, Karen Simonyan, Oriol Vinyals, Alex Graves, Nal Kalchbrenner, Andrew W. Senior, and Koray Kavukcuoglu. 2016. WaveNet: A Generative Model for Raw Audio. CoRR abs/1609.03499 (2016). arXiv:1609.03499 http://arxiv.org/abs/1609.03499

[23] Petra Wagner, Jonas Beskow, Simon Betz, Jens Edlund, Joakim Gustafson, Gustav Eje Henter, Sébastien Le Maguer, Zofia Malisz, Éva Székely, Christina Tånnander, et al. 2019. Speech Synthesis Evaluation-State-of-the-Art Assessment and Suggestion for a Novel Research Program. In Speech Synthesis Workshop (SSW).

[24] Mark West, Rebecca Kraut, and Han Ei Chew. 2019. I'd blush if I could: closing gender divides in digital skills through education. Technical Report. UNESCO. https://unesdoc.unesco.org/ark:/48223/pf0000367416.page=1 